\title{
Distinct Morphological Stages of Dentate Granule Neuron Maturation in the Adult Mouse Hippocampus
}

\author{
Chunmei Zhao, E. Matthew Teng, Robert G. Summers Jr, Guo-li Ming, and Fred H. Gage \\ Laboratory of Genetics, Salk Institute for Biological Studies, La Jolla, California 92037
}

\begin{abstract}
Adult neurogenesis in the dentate gyrus may contribute to hippocampus-dependent functions, yet little is known about when and how newborn neurons are functional because of limited information about the time course of their connectivity. By using retrovirus-mediated gene transduction, we followed the dendritic and axonal growth of adult-born neurons in the mouse dentate gyrus and identified distinct morphological stages that may indicate different levels of connectivity. Axonal projections of newborn neurons reach the CA3 area $10-11$ $\mathrm{d}$ after viral infection, 5- $6 \mathrm{~d}$ before the first spines are formed. Quantitative analyses show that the peak of spine growth occurs during the first 3-4 weeks, but further structural modifications of newborn neurons take place for months. Moreover, the morphological maturation is differentially affected by age and experience, as shown by comparisons between adult and postnatal brains and between housing conditions. Our study reveals the key morphological transitions of newborn granule neurons during their course of maturation.
\end{abstract}

Key words: neurogenesis; spines; mushroom spines; spine motility; neuronal development; plasticity

\section{Introduction}

Neurogenesis occurs in the dentate gyrus throughout adult life in almost all mammals that have been examined (Altman and Das, 1965; Eriksson et al., 1998; Gould et al., 1999). The dentate gyrus, the hippocampus proper, and the subiculum constitute the hippocampal formation, which is critical for certain forms of learning and memory (Squire and Zola-Morgan, 1991). A positive correlation has been established between neurogenesis in the dentate gyrus and an animal's performance on behavioral tasks (Kempermann et al., 1997; van Praag et al., 1999). Ablation of adult neurogenesis through antimitotic drug treatment or localized x-ray irradiation impairs different hippocampus-dependent learning tasks (Shors et al., 2001; Snyder et al., 2005). Although the possibility of nonspecific effects from these treatments has yet to be ruled out (Dupret et al., 2005), these studies suggest that neurogenesis in the dentate gyrus contributes to hippocampusdependent learning and memory; however, it is unknown precisely when new neurons become functional and what specific forms of learning and memory might require adult neurogenesis.

To understand how newborn neurons might contribute to

Received Aug. 27, 2005; revised 0ct. 23, 2005; accepted 0ct. 29, 2005.

This work was supported by grants from the National Institutes of Health-National Institute of Neurological Disorders and Stroke (NS050217 and NS052842) and the Pritzker Neurogenesis Consortium. C.Z. was supported by a postdoctoral fellowship from the Epilepsy Foundation through the generous support of the American Epilepsy Society and the Milken Family Foundation. We thank Gerald Pao for help with the CAG-GFP vector. We thank Ayumu Tashiro, Henriette van Praag, Brad Aimone, Sebastian Jessberger, and Emily Mathews for their critical comments on this manuscript; Henriette van Praag and Wei Deng for help with statistical analyses; Mary Lynn Gage for editorial comments; and Jamie Simon for help with the illustration.

Correspondence should be addressed to Fred H. Gage, Laboratory of Genetics, Salk Institute for Biological Studies, 10010 North Torrey Pines Road, La Jolla, CA 92037. E-mail: gage@salk.edu.

G.I. Ming's present address: Institute for Cell Engineering, Department of Neurology, Johns Hopkins University School of Medicine, 733 North Broadway, BRB 729, Baltimore, MD 21205.

DOI:10.1523/JNEUROSCI.3648-05.2006

Copyright $\odot 2006$ Society for Neuroscience $\quad$ 0270-6474/06/260003-09\$15.00/0 hippocampal functions, it is critical to know the anatomical connectivity of newborn neurons during their course of maturation. The current knowledge of newborn granule neuron development has come mostly from the electrophysiological and morphological characterization of cells randomly selected from the subgranular zone of the dentate gyrus (Wang et al., 2000; Ambrogini et al., 2004; Schmidt-Hieber et al., 2004) and of fluorescently labeled cells in specific transgenic mouse lines (Overstreet et al., 2004; Wang et al., 2005). Although distinct electrophysiological and morphological features of immature cells have been identified through these studies, their timing and duration are not clear. Furthermore, the information obtained is limited to the time window when newly generated cells express the immature neuronal markers or the corresponding transgenes.

The application of retrovirus-mediated gene transfer has made it possible to examine newborn neurons in electrophysiological and morphological details throughout their lifetime (van Praag et al., 2002). Because the connectivity of a neuron is determined mostly by its dendritic-axonal arborization and synaptic contacts, a temporal analysis of the morphological development of newborn granule neurons will provide insights into when these cells can integrate into the existing hippocampal circuit. Here, we describe the morphogenesis of newly generated dentate granule neurons in the adult mouse hippocampus. New neurons were labeled with green fluorescent protein (GFP) through stereotaxic delivery of the retrovirus CAG-GFP in which the CAG promoter consists of CMV enhancers, chicken $\beta$-actin promoter, and a large synthetic intron (Kootstra et al., 2003). We focused on the dendritic and axonal outgrowth to characterize the initial stages of neuronal maturation. To describe the later stages of maturation, we analyzed the developmental changes in dendritic spines. The age-dependent maturation was examined by comparing neurons born in the adult and postnatal brains, and the regulatory role of the animal's experience was studied by housing mice with unlimited access to running wheels. 


\section{Materials and Methods}

Retrovirus-mediated labeling of new neurons in the mouse hippocampus. The murine Moloney leukemia virus-based retroviral vector CAG-GFP is described in detail in Results; it was a gift from Drs. Gerald Pao and Inder Verma (Salk Institute, La Jolla, CA). The concentrated viral solution $\left(10^{8}\right.$ $\mathrm{pfu} / \mathrm{ml}$ ) was prepared with human embryonic kidney $293 \mathrm{~T}$ as a package cell line and collected through ultra-speed centrifugation. The virus solution was delivered to the dentate gyrus of the mouse hippocampus through stereotaxic surgery as described previously (van Praag et al., 2002). Specifically, the difference $(d)$ between bregma and lambda in anteroposterior coordinates was determined. The injection site for adult mice was calculated as follows by using the position of the bregma as reference: anteroposterior, $-(1 / 2) \times d$ $\mathrm{mm}$; lateral, $-1.6 \mathrm{~mm}$ (if $d \leq 1.6$ ) or $-1.7 \mathrm{~mm}$; ventral, $-1.9 \mathrm{~mm}$ (from dura). The injection site for the postnatal day 10 (P10) pups was determined similarly in anteroposterior and lateral coordinates, with $-1.8 \mathrm{~mm}$ for the ventral coordinate. The mice used for this study were female C57BL/6 and were $7-10$ weeks of age at the time of surgery, unless stated otherwise. They were housed in standard cages (control) or in cages with a running wheel (running), with two to four mice per cage. All animals were coded in this study, and a blinded analysis was used for quantitative comparisons. The animal protocols were approved by the Salk Institutional Animal Care and Use Committee.

Sample preparation for morphological analyses and immunohistochemistry. Experimental animals were anesthetized with a mixture of ketamine/xylazine $(100 \mathrm{mg} / \mathrm{kg}, 10 \mathrm{mg} / \mathrm{kg})$ and perfused transcardially with $0.9 \%$ saline followed by $4 \%$ paraformaldehyde. The brain samples were postfixed with $4 \%$ paraformaldehyde and equilibrated in 30\% sucrose. Coronal sections of $40 \mu \mathrm{m}$ were prepared with a sliding microtome. Brain sections of one-in-six series were selected for immunostaining. The following antibodies were used: rabbit anti-GFP (Invitrogen, Eugene, $\mathrm{OR}$ ), mouse anti-neuronal-specific nuclear protein (NeuN) (Chemicon, Temecula, CA), rabbit anti-Ki67 (Novocastra Laboratories, Newcastle, UK), FITC- or cyanine 3-conjugated donkey anti-rabbit and cyanine 3-conjugated donkey anti-mouse antibodies (Jackson ImmunoResearch, West Grove, PA). GFP staining was used only for the assessment of axonal growth. $4^{\prime} 6^{\prime}$-Diamidino-2-phenylindole (DAPI) was used to reveal nuclei. For dendritic growth and all spine analyses, GFP+ neurons were identified by visual assessment of their morphology.

Dendritic and axonal growth of GFP + neurons. For dendritic growth analysis, z-series at $1 \mu \mathrm{m}$ intervals were acquired with a $40 \times$ oil lens with a Bio-Rad (Hercules, CA) Radiance 2100 confocal system. Twodimensional maximum intensity projections of each z-series were created with the Confocal Assistance Program (Bio-Rad) in the BMP (bitmap) format, and the files were then imported into IGL Trace (http://synapses.bu.edu/tools/trace/trace.htm) to measure dendritic length. The number of branching points was counted manually from the same images. A total of 5-10 cells from two mice were analyzed for each data point. For the qualitative assessment of axonal growth, survey z-series of the area of interest were taken with a $20 \times$ oil lens at $1 \mu \mathrm{m}$ intervals. For the quantitative measurement of axonal growth, a Nikon (Melville, NY) E800 microscope connected to a CCD camera (Spot RT; Diagnostic Instruments, Sterling Heights, MI) was used to image the hippocampal area. The growth of axonal fibers was determined by tracing the border between stratum pyramidale and stratum radiatum, starting from the crossing point between pyramidale (determined by NeuN staining) and a straight line drawn connecting the ends of the two blades of the dentate gyrus (supplemental Fig. $1 a$, available at www.jneurosci.org as supplemental material). A total of 6-12 sections from two or more mice were used for each time point, except for $11 \mathrm{~d}$ postinfection (dpi) (four sections from one mouse).

Spine density and classification of mushroom spines. Images of GFPlabeled dendritic processes at the outer molecular layer were acquired at $0.5 \mu \mathrm{m}$ intervals with the Bio-Rad R2100 confocal system with a plane apochromatic $60 \times$ oil lens [numerical aperture (NA), 1.4; Nikon] and a digital zoom of 4 . The Bio-Rad image files were subjected to five iterations of deconvolution with the AutoDeblur program (AutoQuant, Troy, NY). Maximum intensity projections of $\mathrm{z}$-series were created with the Confocal Assistance Program in BMP format, and the files were then imported into IGL Trace. The length of each dendritic segment was determined by tracing the center of the dendritic shaft, and the number of spines was counted manually from the two-dimensional projections. The linear spine density was calculated by dividing the total number of spines by the length of the dendritic segment.

For classification of mushroom spines, major and minor axes of each spine head were identified, and the size of the spine head was represented by the estimated surface area with the function $1 / 4 \times \pi \times D_{\text {major }} \times D_{\text {minor }}$ (supplemental Fig. $2 a$, available at www.jneurosci.org as supplemental material). The estimated surface areas of $>100$ spines were compared with the actual measured surface areas with the IGL Trace program, and the correlation value was $>0.97$ (supplemental Fig. $2 b$, available at www.jneurosci.org as supplemental material). The threshold value for mushroom spine was set at $0.4 \mu \mathrm{m}^{2}$, which is close to the value of [average $+2 \times \mathrm{SD}$ ] calculated from $>400$ spines derived from three individual dendritic segments selected randomly from control samples at 126 dpi (supplemental Fig. $2 c$, available at www.jneurosci.org as supplemental material). Relatively large spines were then selected by visual assessment, and their major and minor axes were measured three times independently with the IGL Trace program. A spine was considered of mushroom type if the average estimated area from three measurements was $\geq 0.4 \mu \mathrm{m}^{2}$.

For the final quantification, a total of 38 or more dendritic segments from three to four mice were used at 28,56 , and $126 \mathrm{dpi}$, and 24 or more segments from two mice were used at $21 \mathrm{dpi}$. Confocal imaging and data quantification were done by the same person, who was blinded to the experimental conditions.

Sample preparation for spine motility analysis. Acute brain slices were prepared as described previously (van Praag et al., 2002). After recovery in artificial CSF solution for $\geq 30 \mathrm{~min}$, the brain slices were transferred to either the closed-dish (FCS2; Bioptechs, Butler, PA) or open-dish temperaturecontrolled chamber (Delta T4 Culture dish system; Bioptechs) and perfused continuously with oxygenated artificial CSF solution at $30^{\circ} \mathrm{C}$. The system was mounted on an inverted Nikon TE2000 microscope or an upright Olympus BX51 (Olympus, Melville, NY) connected to the Bio-Rad R2100 confocal system for time-lapse imaging. For imaging with the inverted microscope, number 1 coverslips and a $60 \times$ water immersion lens (NA, 1.25; Nikon) were used to maximize the working distance. The $60 \times$ water immersion (dipping) lens (NA, 0.9; Olympus) was used for the upright microscope. Images of dendritic processes identified at the outer molecular layer were taken every 15 or $30 \mathrm{~min}$ for $2 \mathrm{~h}$, and four time points at $30 \mathrm{~min}$ intervals for the first $90 \mathrm{~min}$ were selected for quantitative analyses. The image files were subjected to five iterations of deconvolution (AutoDeblur; AutoQuant, Watervliet, NY), and the image stacks were aligned with the alignment function (Image-Pro, Media Cybernetics, Silver Spring, MD). Maximum intensity projections were created with Image-Pro for each time point. An image stack was then created for these four time points, aligned with Image-Pro software, and further aligned manually by means of Photoshop with the dendritic shaft as reference.

Quantification of spine motility. Spine motility is quantified by using the "measure colocalization" function of MetaMorph software (Molecular Devices, Sunnyvale, CA). Spines were first cropped out of the original time series (supplementary Fig. 3a,b, available at www.jneurosci.org as supplemental material). The motility of each spine was determined as follows: $\Sigma_{(1-3)}$ nonoverlapping areas/average areas/3 (supplemental Fig. $3 c$, available at www.jneurosci.org as supplemental material). Not all spines were analyzed. A person blinded to the experimental conditions was asked to select spines from the first and last time frames. The spines were selected on the basis of the condition that they were reasonably set apart from neighboring spines to minimize noise in the measurement. All data were used for the final quantification even if the noise was high for some of the spines. A total of 100 or more individual spines from four to eight time series was used in the final quantification for each time point.

Statistics and data presentation. All statistical analyses were done with Statview. One-way ANOVA was used to compare axonal growth, spine density, mushroom spine density, and spine motility at different time points. Fisher's PLSD was used for post hoc analysis when a significant difference was found with ANOVA. Unpaired two-tailed $t$ test was used for other comparisons, including dendritic growth and branching and comparisons between control and running groups at different time points. All data are presented as mean \pm SEM. 

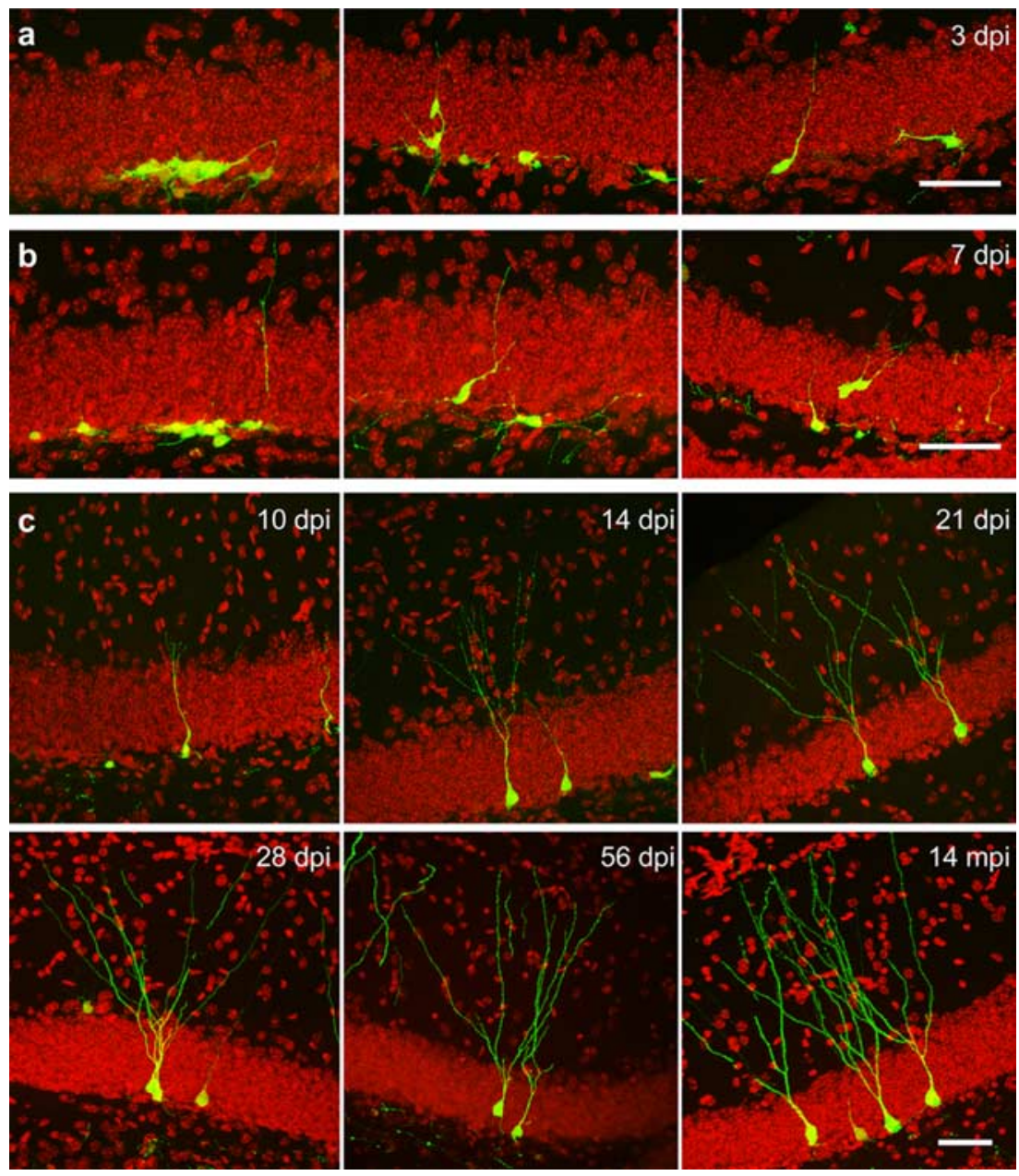

Figure 1. The morphological development of neurons born in the adult mouse brain. Dividing cells in the dentate gyrus of 7 to 10-week-old C57BL/6 mice were labeled with GFP through retrovirus-mediated gene transduction. Mice were killed at different time points as indicated. Images are oriented with the molecular layer on the upside. $\boldsymbol{a}$, Cells at $3 \mathrm{dpi}$. $\boldsymbol{b}$, Cells at $7 \mathrm{dpi}$. c, Cells at 10, $14,21,28$, and $56 \mathrm{dpi}$, and also $14 \mathrm{mpi}$. Scale bars, $50 \mu \mathrm{m}$. DAPl is red; GFP is green.

\section{Results \\ Efficient labeling of adult-born neurons with retroviral vector CAG-GFP}

In this study, we used an improved retroviral vector CAG-GFP to label newly generated granule neurons in the adult mouse hippocampus through stereotaxic surgery (supplemental Fig. $4 a$, available at www.jneurosci.org as supplemental material) (van Praag et al., 2002). CAG-GFP uses the backbone of pCLNCXv.2, and the expression of GFP is driven by the compound promoter CAG (Naviaux et al., 1996). This vector also contains the woodchuck hepatitis virus posttranscriptional element, which reportedly increases protein production by stabilizing the transcript (Zufferey et al., 1999). The number of newborn neurons labeled with CAG-GFP is much higher than that with the previous vector NIT-GFP (van Praag et al., 2002, 2005), and the fluorescent intensity of labeled neurons is also stronger. Both vectors are replication incompetent, so only dividing cells at the time of surgery can be infected. Consistent with our previous findings (van Praag et al., 2002), GFP-labeled (GFP+) neurons exhibit elaborate and spiny dendritic trees 4 weeks after viral transduction (Figs. 1, 2a, $28 \mathrm{dpi}$ ) (van Praag et al., 2002). Furthermore, the expression of GFP in labeled cells is persistent, even at 14 months postinfection (mpi).

\section{Elaborate dendritic arborization of} newborn cells within 3 weeks

To study the morphogenesis of new neurons in the adult, we delivered concentrated virus $\left(10^{8} \mathrm{pfu} / \mathrm{ml}\right)$ into the dentate gyrus of 7 - to 10-week-old female C57BL/6 mice. These mice were killed at different time points after viral injection (supplemental Fig. $4 b$, available at www.jneurosci. org as supplemental material). By using Ki67 as a marker of proliferating cells, we quantified the number of GFP + cells that were still dividing at 3, 7, 10, and 28 dpi. The fraction of $\mathrm{Ki} 67+\mathrm{GFP}+$ cells decreased from $18.3 \pm 6.5 \%$ at 3 dpi to $3.5 \pm$ $3.5 \%$ at $7 \mathrm{dpi}, 0.9 \pm 0.9 \%$ at $10 \mathrm{dpi}$, and $1.2 \pm 0.7 \%$ at $28 \mathrm{dpi}$. A significant difference $\left(F_{(3,9)}=5.64 ; p<0.02\right.$; ANOVA $)$ was found among groups. Post hoc comparisons showed that 3 dpi was different from the other three time points $(p<0.02$; Fisher's PLSD). This finding suggested that most of the GFP + cells analyzed were born shortly after viral infection; therefore, we used the time of virus infection as reference in this study.

Most of the GFP + cells were located at the hilar border of the granule cell layer at $3 \mathrm{dpi}$ and at all later time points examined, even at 14 mpi (Fig. 1). A small portion of the cells migrated to the middle third and less frequently to the outer third of the granule cell layer, and they could be seen in these positions as early as $3 \mathrm{dpi}$ (Fig. $1 a$, middle panel, 3 dpi; Fig. $1 b$, right panel, 7 dpi). GFP + cells were sometimes found in clusters at 3 and 7 dpi (Fig. $1 a, b$, left panels); some bore processes that were parallel to the granule cell layer (Fig. $1 a, b$ ). Occasionally, cells with processes spanning the granule cell layer were found at $3 \mathrm{dpi}$; these cells were more commonly seen at $7 \mathrm{dpi}$ (Fig. 1a,b, right panels). As was found with bromodeoxyuridine (BrdU) labeling (van Praag et al., 1999), $71.7 \pm 8.2 \%$ of GFP + cells were of neuronal phenotype, which was determined by the coexpression of the neuronal marker NeuN at 28 dpi.

Some GFP + cells at 10 and 14 dpi started to resemble the typical morphology of mature granule neurons, although they still had the characteristics of immature neurons. Their dendritic processes were short, and varicosities were on their dendritic processes (Figs. 1c, 2a) (Jones et al., 2003). The apical dendrites of $\mathrm{GFP}+$ neurons reached the inner molecular layer at $10 \mathrm{dpi}$, and their arborization appeared more elaborate than that at $7 \mathrm{dpi}$. The dendritic processes grew to the middle of the molecular layer at $\sim 14$ dpi and reached the edge of the molecular layer in some of the neurons at $21 \mathrm{dpi}$. The more mature-looking neurons at 21 dpi had elaborate dendritic arborization, and their overall morphology resembled neurons at later time points (Fig. 1c, 21, 28, and $56 \mathrm{dpi}, 14 \mathrm{mpi}$ ).

\section{Delayed maturation of granule neurons in the adult brain}

Electrophysiological and morphological studies have suggested that granule neurons go through similar transitions in 
the neonatal and adult brains (Liu et al., 1996; Ambrogini et al., 2004). For example, there is a switch from GABAergic to glutamatergic excitatory input to immature granule neurons, but it is not clear whether these transitions follow a similar time course in neonatal and adult brains. In addition, we were curious about whether neurons born in the adult brain were morphologically different from those born during early development; therefore, we compared granule neuron development in the adult and postnatal brains. P10 was chosen for this initial study because it is within the peak period of ongoing postnatal neurogenesis.

Because dendritic spines are the major postsynaptic sites of granule neurons to receive glutamatergic inputs, the timing of spine formation may reflect one of the important physiological transitions. For neurons born in the adult brain, dendritic spines are clearly present at $21 \mathrm{dpi}$ but not at $14 \mathrm{dpi}$ (Fig. 2a). To determine precisely the initial timing of spine growth, we examined cells at 15-17 dpi. Spines were seen at $17 \mathrm{dpi}$, although only in a small portion of the cells ( 2 of 11 cells examined). Spines could also be found at 16 dpi but not at 15 dpi (Fig. $2 b$ ). These observations suggest that spine formation starts at $\sim 16 \mathrm{dpi}$ in granule neurons born in the adult mouse brain.

GFP + neurons labeled at P10 clearly showed dendritic spines at $14 \mathrm{dpi}$, suggesting that spine formation started earlier in the postnatal brain (data not shown). We subsequently examined cells at 10-13 dpi. Spines were present at 12 and 13 dpi but not at 10 or $11 \mathrm{dpi}$ (Fig. $2 c$ ); therefore, we concluded that the initiation of spine growth in newborn granule neurons was delayed for $\sim 4 \mathrm{~d}$ in the adult mouse brain.

It is not clear what triggers the initiation of spine growth. One possibility is that spine growth is related to dendritic growth. At the time that spine growth is initiated, almost all spiny neurons have their processes in the outer third of the molecular layer (data not shown). This finding suggests that the delay in spine growth may result from a delay in dendritic growth.

To quantify dendritic growth, we scored dendritic length and branching points. We chose more mature-looking cells for this analysis, to avoid putative influences from progenitor cell cycle length and frequency. These measurements underestimated the total dendritic length and branching points because sections of $40 \mu \mathrm{m}$ thickness were used. We compared neurons at $12 \mathrm{dpi}$, when spines are first found for neurons born at P10. Supporting our hypothesis, the dendritic length of 12 dpi adult neurons was significantly less than that of 12 dpi P10 neurons, even when we used the less mature, spineless $12 \mathrm{dpi}$ P10 neurons for this comparison (Fig. $2 d, e)\left(t_{(13)}=3.29\right.$; $\left.p<0.006\right)$.

C

e as mean \pm SEM in $\boldsymbol{e}$ and $\boldsymbol{f}$.
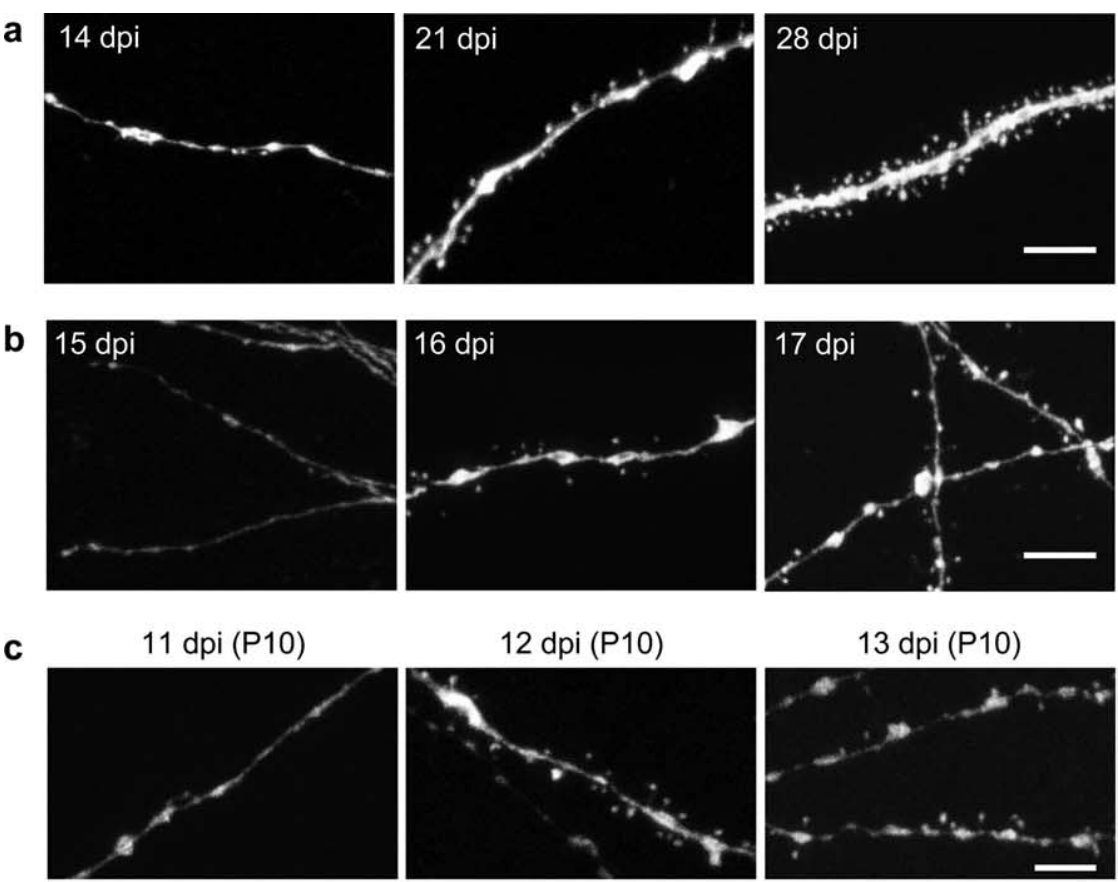

12 dpi (P10)

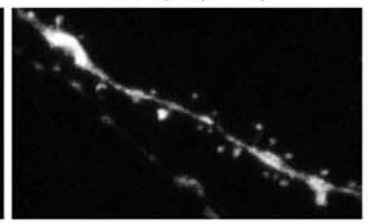

13 dpi (P10)
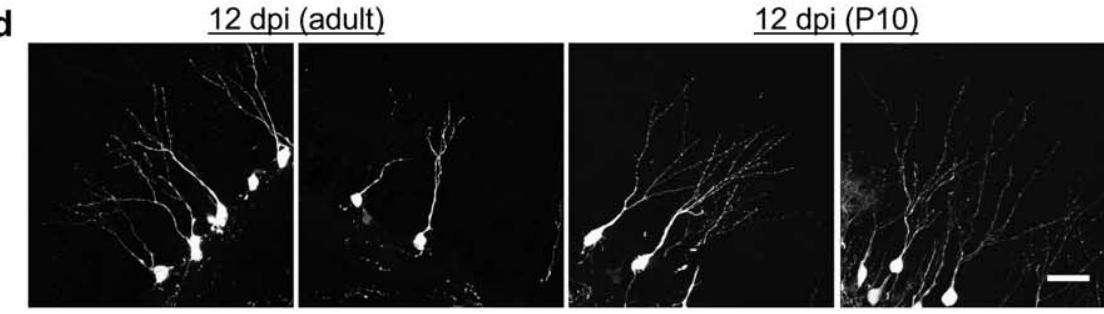

\section{f}
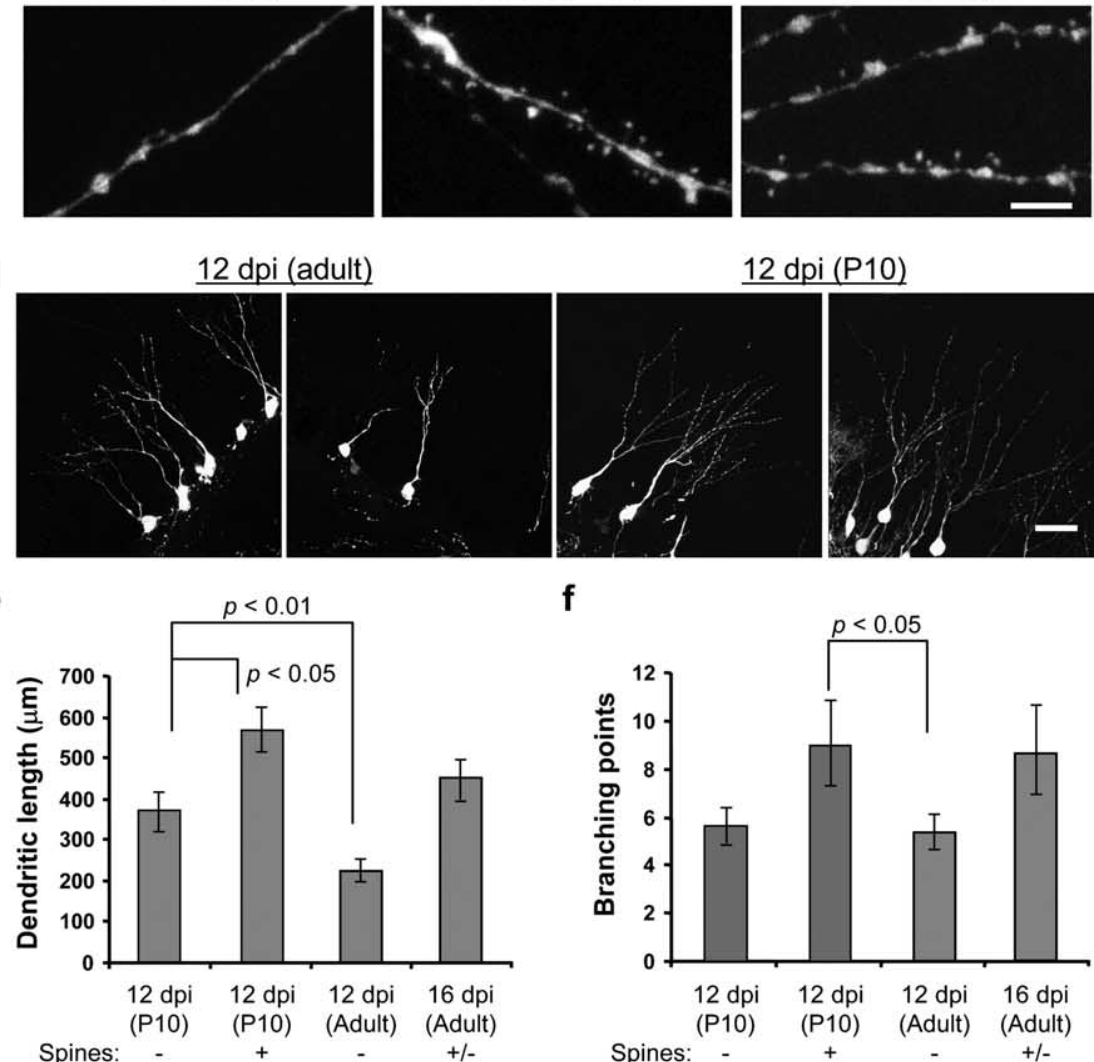

Figure 2. The initiation of spine genesis in newly generated granule neurons. $\boldsymbol{a}$, Examples of dendritic segments from mouse brains taken at 14, 21, and $28 \mathrm{dpi}$. Scale bar, $5 \mu \mathrm{m}$. $\boldsymbol{b}$, Examples of dendritic segments at 15, 16, and $17 \mathrm{dpi}$. Scale bar, $5 \mu \mathrm{m}$. $\boldsymbol{C}$, Examples of dendritic segments of neurons born at P10. Spines start to grow before $12 \mathrm{dpi}$ (middle panel). Scale bar, $5 \mu \mathrm{m}$. $\boldsymbol{d}$, Representative images of more mature-looking cells at $12 \mathrm{dpi}$. Scale bar, $50 \mu \mathrm{m}$. $\boldsymbol{e}$, Quantification of dendritic length of cells born in the adult brain at 12 and $16 \mathrm{dpi}$, as well as cells born at P10 at $12 \mathrm{dpi}$. $\mathrm{f}$, Quantification of branching points. Data are presented

The dendritic length of spine-bearing cells was significantly longer than that of spineless cells in the same brains [Fig. $2 e, 12$ dpi $(\mathrm{P} 10)]\left(t_{(8)}=3.04 ; p<0.02\right)$. We also examined the maturelooking cells born in the adult brain at $16 \mathrm{dpi}$, when spines are first seen in the adult-born cells. The dendritic complexity of 16 dpi adult neurons was similar to that of the 12 dpi P10 neurons, because we did not detect any significant difference in dendritic length or the number of branching points [Fig. $2 e$, $12 \mathrm{dpi}$ (P10), $16 \mathrm{dpi}$ (Adult)]. In addition, the $12 \mathrm{dpi}$ P10 spiny neurons had more branching points than the $12 \mathrm{dpi}$ adult 

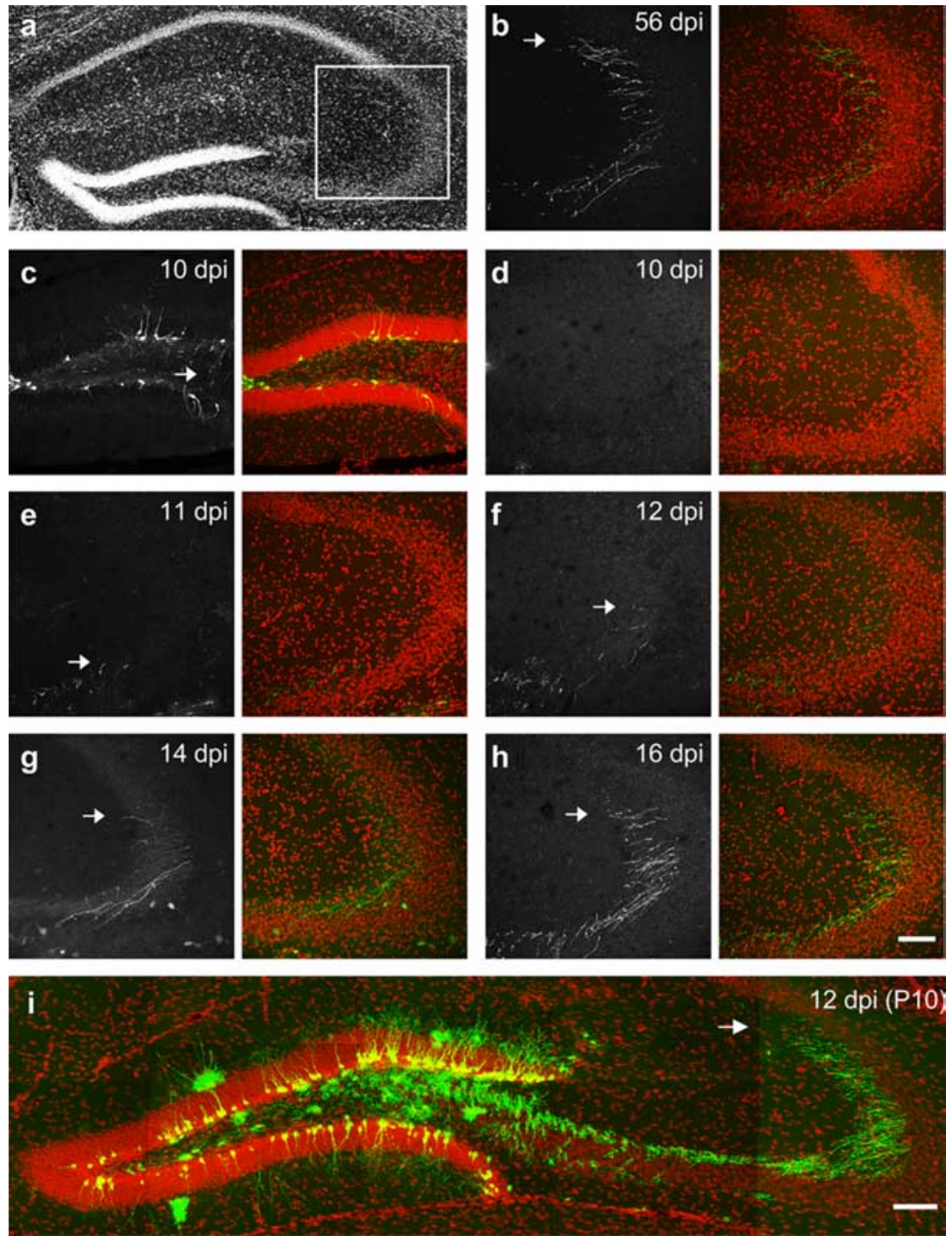

Figure 3. Axonal growth in newborn granule neurons. Sections of similar levels in the anterior-posterior axis were imaged for axonal growth in the adult $(\boldsymbol{a}-\boldsymbol{h})$. Arrows indicate the end of the fibers. DAPI is red; GFP is green. Scale bar, $100 \mu \mathrm{m}$. GFP signal in $\boldsymbol{c}-\boldsymbol{h}$ was enhanced by GFP antibody staining. $\boldsymbol{a}$, The standard hippocampal section used for all time points. The square indicates the CA 3 area imaged in $\boldsymbol{b}$ and $\boldsymbol{d}-\boldsymbol{h} . \boldsymbol{b}$, Axonal fibers at 56 dpi were used as reference. $\boldsymbol{c}$, The labeling at $10 \mathrm{dpi}$ in the dentate gyrus, showing that the lack of GFP in $\boldsymbol{d}$ is not caused by a lack of labeling. Thin fibers are clearly seen in the hilus (arrow). $\boldsymbol{d}-\boldsymbol{h}$, Axonal fibers in the CA3 area at 10,11, 12, 14, and 16 dpi. $\boldsymbol{i}$, Axonal growth of neurons labeled at P10.

neurons (Fig. 2f) $\left(t_{(13)}=2.51 ; p<0.03\right)$. These data suggest that there is indeed a delay in dendritic growth in neurons born in the adult and indicate a positive correlation between dendritic complexity and the initiation of spine growth in newborn neurons.

\section{Axonal fibers reach CA3 before the formation of the first spines}

The connectivity of a neuron is determined by both afferent input through dendrites and efferent output through axons. Previously, retrograde tracing studies showed that new neurons send their axon projections into the CA3 area (Markakis and Gage, 1999); this process can be detected as early as $10 \mathrm{~d}$ after cells are born in the adult rat brain (Hastings and Gould, 1999); however, it is not known precisely when axonal fibers of new neurons reach CA3 and how axonal and dendritic growth are coordinated.

To determine the timing of axonal growth relative to dendritic growth, we examined the appearance of axon fibers from granule neurons born in the adult mice. For all time points studied, we focused on sections at similar anteroposterior positions (Fig. $3 a$ ), and the GFP fluorescence in the CA3 area was imaged. A sample at 56 dpi was used as reference (Fig. 3b). We started from 16 dpi (Fig. 3h) because this is the earliest time when spines were found. The axonal fibers at 16 dpi had not migrated as far as those at 56 dpi (Fig. 3, compare $b, h$, arrows), suggesting they continued to grow after $16 \mathrm{dpi}$. Nevertheless, they had clearly reached the CA3 area and were quite distal to the dentate; therefore, we decided to trace axonal growth to earlier time points until $10 \mathrm{dpi}$, when no GFP + fibers were found in the area imaged (Fig. $3 d$ ). Figure $3 c$ shows the GFP labeling in the dentate gyrus, and thin fibers can be seen in the hilus at $10 \mathrm{dpi}$ (arrow). Images from samples at 11-16 dpi depict the growth of axonal fibers, which project farther and farther into the CA3 region (Fig. $3 e--h$, arrows).

We then scored the overall axonal growth at $7,10,11,12,14,16$, and $56 \mathrm{dpi}$. Because it was difficult to trace individual axons, we chose to measure the farthest length of axonal growth in the sections sampled (Materials and Methods) (supplemental Fig. 1, available at www. jneurosci.org as supplemental material). The traced lengths were $-87 \pm 39,137 \pm$ $70,373 \pm 70,478 \pm 142,750 \pm 126$, $1146 \pm 43$, and $1339 \pm 94 \mu \mathrm{m}$ for each time point, respectively (supplemental Fig. $1 b$, available at www.jneurosci.org as supplemental material). A significant difference $\left(F_{(6,45)}=30.9 ; p<0.0001\right.$; ANOVA $)$ was found among groups. Post hoc comparisons showed significant differences among most of the groups $(p<0.02)$. No significant differences $(p>0.05)$ were found between 7 and $10 \mathrm{dpi}, 10$ and $11 \mathrm{dpi}$, 11 and $12 \mathrm{dpi}, 12$ and $14 \mathrm{dpi}$, or 16 and 56 dpi. With these observations, we conclude that axonal and dendritic outgrowth are concomitant and that axons reach the CA3 before the first spines are formed on newborn neurons.

This finding is also true for neurons born at P10. Axonal fibers were abundant in the distal CA3 area at $12 \mathrm{dpi}$ when spines were first detected in these cells (Fig. 3i). Similar to dendritic growth, axonal growth was also delayed in neurons born in the adult. The mossy fiber axons of 12 dpi P10 neurons had migrated to positions similar to those of $16 \mathrm{dpi}$ adult neurons (Fig. $3 h, i)$ and were much more distal to the dentate than 12 dpi adult neurons (Fig. $3 f)$. It remains to be determined whether the axons made functional synapses with CA3 pyramidal neurons at this time point.

\section{Prolonged morphological plasticity of dendritic spines in} newborn neurons

Although the overall morphology of GFP + neurons resembled that of typical mature granule neurons at $28 \mathrm{dpi}$, they were still 
different from mature cells in fine dendritic morphology, because their spine density had not reached the level of 4-month-old cells (van Praag et al., 2002). Because mature granule neurons receive most of their synaptic inputs through dendritic spines, the number and shape of the dendritic spines are indicative of the connectivity of these cells. To analyze the development of dendritic spines of newborn neurons, we focused on three features of spines: density, shape, and motility.

As mentioned previously, spine growth starts at $\sim 16 \mathrm{dpi}$ in the adult brain. Spine density at 21, 28, 42, 56, and 126 dpi was quantified, and a significant difference $\left(F_{(4,191)}=138 ; p<0.0001\right.$; ANOVA $)$ was found among different time points (Fig. $4 b$, blue). Post hoc analysis showed that significant differences existed among all time points $(p<0.02)$ except between 56 and 126 dpi $(p>0.1)$. There was a sharp increase in spine density between 21 and $28 \mathrm{dpi}$, when the average spine density changed from $0.43 \pm 0.09$ to $1.95 \pm 0.08$ spines per micrometer (Fig. $4 b$, blue). Spine density continued to increase until $56 \mathrm{dpi}(2.61 \pm 0.07$ spines per micrometer; $56 \mathrm{dpi}$ ) when it reached a plateau (Fig. $4 b$, blue).

Spines are extremely diversified (Jones and Powell, 1969; Peters and KaisermanAbramof, 1970), and they have been categorized into four different types on the basis of their morphology: mushroom, thin, stubby, and filopodia (Nimchinsky et al., 2002; Sala, 2002). Filopodia and stubby spines are often associated with neurons during early development, whereas thin and mushroom spines are more abundant in mature neurons (Nimchinsky et al., 2002). The numbers of filopodial protrusions (long and thin protrusions with no enlarged head) and stubby spines (spines with no neck) were low in all samples examined in this study, including the samples taken when the earliest spines were found (Fig. 2). Most of the spines that we observed were thin spines that had small spine heads and thin necks (Figs. 2, 4a).

It has been suggested that the size of the spine head is correlated with the area of postsynaptic density, the presynaptic parameters such as active zone size and number of docked vesicles, and the efficacy of the corresponding synapse (Harris and Stevens, 1989; Schikorski and Stevens, 1997; Matsuzaki et al., 2001); therefore, we focused on the mushroom spines, which are named for the large size of their spine head (Nimchinsky et al., 2002; Sala, 2002) and are likely to be associated with strong synapses. The density of mushroom spines varies greatly among different neurons, even among those from the same animal (Fig. $4 c$, blue). A significant difference in mushroom spine density was found among different time points $\left(F_{(4,224)}=11.8 ; p<0.0001\right)$. Post hoc analysis showed that mushroom spine densities at 21 and 126 dpi were significantly different from all other time points $(p<0.01)$. No differences were found among 28,42 , and $56 \mathrm{dpi}$ $(p>0.2)$. Similar to protrusion density, there was an initial sharp increase in mushroom spine density between 21 and $28 \mathrm{dpi}$, and the rate of increase slowed down afterward. In contrast to the
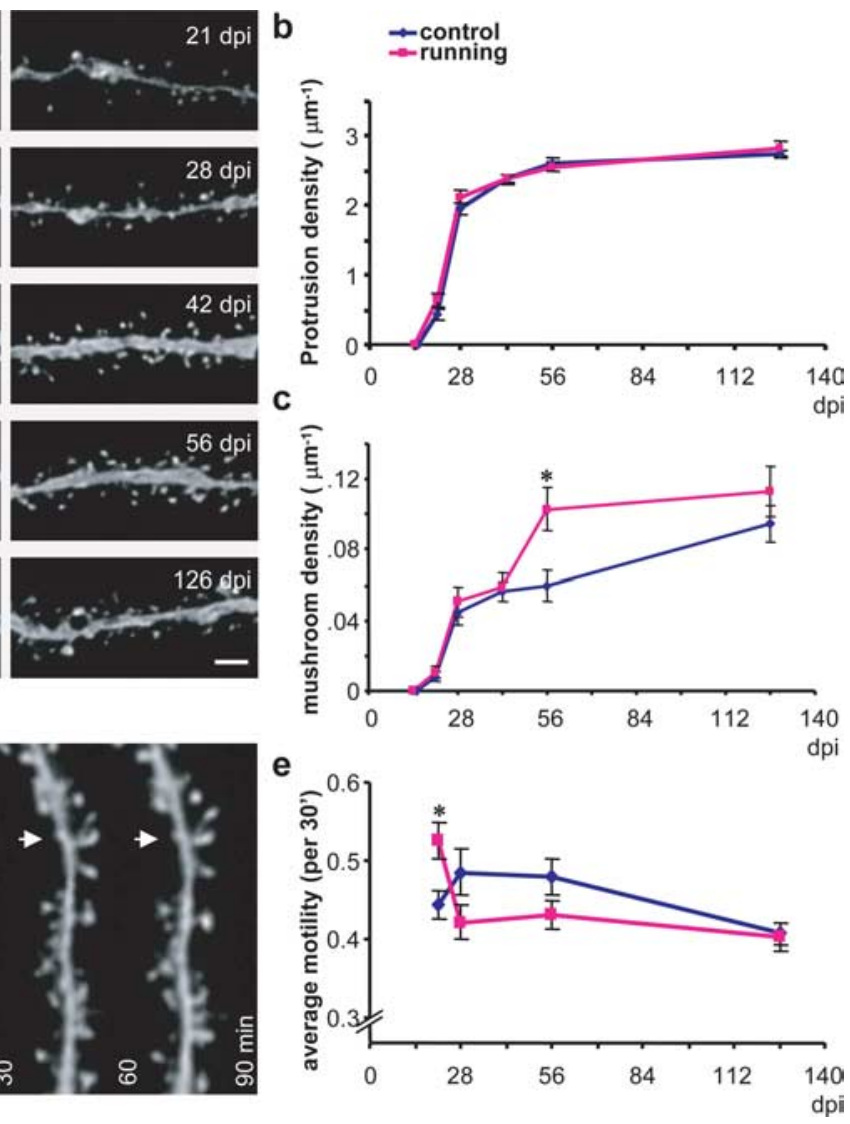

dpi

Figure 4. The maturation of granule neurons born in the adult mouse brain: dendritic spine analyses. $\boldsymbol{a}$, Representative images of dendritic segments from newborn neurons at 21, 28, 42, 56, and $126 \mathrm{~d}$ after viral infection. $\boldsymbol{b}$, Quantification of total protrusion density. 作 retractions of a spine over the time series. $\boldsymbol{e}$, Quantification of spine motility. Data for $\boldsymbol{b}, \boldsymbol{c}$, and $\boldsymbol{e}$ are presented as mean $\pm \mathrm{SEM}$. The asterisks indicate where significant differences were seen between control and running mice $(p<0.01)$. Scale bars, $2 \mu \mathrm{m}$.

relatively stable protrusion density after $56 \mathrm{dpi}$, mushroom spine density continued to increase. It was significantly higher at 126 $\mathrm{dpi}\left(0.095 \pm 0.01 \mu \mathrm{m}^{-1}\right)$ than at $56 \mathrm{dpi}\left(0.059 \pm 0.008 \mu \mathrm{m}^{-1}\right.$; $p<0.01$ ) (Fig. $4 c$, blue). This finding indicates a prolonged structural plasticity of newborn neurons.

Spines are dynamic, and spine motility adds to the structural plasticity of the nervous system (Bonhoeffer and Yuste, 2002). Spine motility describes any movements of spines, including growth, retraction, and changes in size, shape, and position. The average motility of all protrusions was examined with time-lapse imaging of acute brain slices (Fig. 4d) (supplemental Fig. 3, available at www. jneurosci.org as supplemental material) (Materials and Methods). Motile spines were observed throughout all time points (Fig. $4 d$, arrow) (supplemental videos, available at www.jneurosci.org as supplemental material). Spine motility at different time points was quantified, and a significant difference was found between groups $\left(F_{(3,861)}=4.4 ; p<0.005\right)$. Post hoc analysis showed that the average spine motility at 28 and 56 dpi was significantly higher than that at 21 dpi $(p<0.05)$ and $126 \mathrm{dpi}(p<0.01)$ (Fig. 4e, blue).

Accelerating neuronal maturation by voluntary exercise To study the effect of an animal's experience on newborn neuron maturation, we housed mice with unlimited access to running wheels starting $7 \mathrm{~d}$ before surgery until they were killed for exper- 

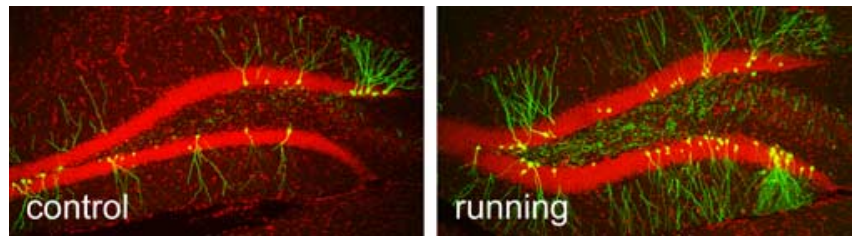

Figure 5. Representative images of GFP labeling in control and running mice at $56 \mathrm{dpi}$.

iments (supplemental Fig. 4b, available at www.jneurosci.org as supplemental material). Consistent with previous studies that used BrdU labeling (van Praag et al., 1999), we observed more $\mathrm{GFP}+$ neurons in running mice than in control mice. For example, the total number of GFP + neurons in running mice was estimated to be $1143 \pm 151,2.5$ times that in control mice ( $456 \pm$ $\left.107 ; t_{(6)}=3.7 ; p<0.01 ; n=4\right)$ at $56 \mathrm{dpi}$ (Fig. 5). These numbers were estimated from the GFP + cell number counted from onein-six series of brain sections. Granule neurons in the running mice showed similar rates of maturation during the initial stages; they were morphologically indistinguishable from those in the control mouse brain (data not shown). The earliest time that we observed spine growth was at $15 \mathrm{dpi}$, which was $1 \mathrm{~d}$ earlier than was seen in the control mice. This $1 \mathrm{~d}$ advancement in spine growth does not seem to be significant, because we did not see a difference in spine density between running and control mice at 21 and 28 dpi or at any other time points that we examined (Fig. $4 b$ ); however, we cannot exclude the possibility that our experimental design is not sensitive enough to detect early differences between control and running during neuronal maturation. For example, running has been shown to increase cellular proliferation, which could lead to the inclusion of more young cells in the final population analyzed in the running group if the labeled cells go through more cell cycles in runners than in controls.

Specific examination of mushroom spines, however, revealed that structural modifications on spines were different under these two housing conditions. Mushroom spine density at different time points was significantly different between groups $\left(F_{(4,189)}=15.3 ; p<0.0001\right)$. Post hoc analysis showed that, in contrast to control conditions, mushroom spine density in runners at 56 dpi was significantly higher than at 28 and $42 \mathrm{dpi}(p<0.002)$ but not different from $126 \mathrm{dpi}(p>0.4)$. Consistently, unpaired two-tailed $t$ tests between the two housing conditions at each time point showed that neurons in running mice had significantly higher mushroom spine density than those in control mice at $56 \mathrm{dpi}$ (Fig. $4 c)(0.1 \pm 0.01$ and $0.059 \pm 0.008 \mu \mathrm{m}^{-1}$ for running and control mice, respectively; $\left.t_{(76)}=2.9 ; p<0.006\right)$. There was no difference in mushroom spine density between the two housing conditions at $126 \mathrm{dpi}\left(t_{(80)}=1.1 ; p>0.25\right)$, suggesting that running is probably accelerating the formation rather than increasing the total number of mushroom spines.

Spine motility of these neurons, specifically during early maturational stages, was also affected by running. ANOVA analysis showed that spine motility was different among time points in running mice $\left(F_{(3,693)}=6.16 ; p<0.001\right)$. Post hoc analysis showed that spine motility was significantly higher at $21 \mathrm{dpi}$ than at later time points $(p<0.005)$. Unpaired $t$ tests between the two housing conditions showed that spine motility was significantly higher in running mice than in controls at $21 \mathrm{dpi}$ (Fig. $4 e)\left(t_{(373)}=\right.$ $3.6 ; p<0.001)$. Spine motility in running mice quickly decreased to the basal level at $28 \mathrm{dpi}$ ( $p<0.005 ;$ post hoc Fisher's PLSD). The time of the highest spine motility in running mice was correlated with the peak of spine genesis (Fig. 4b, $21 \mathrm{dpi}$ ). These data suggest that, in addition to promoting neurogenesis, running also has an effect on newborn neuron maturation that could influence the rate of integration into the hippocampal circuit.

\section{Discussion}

We have described the morphogenetic events of dentate granule neurons born in the adult mouse brain. The maturation of granule neurons can be segregated into four stages (Fig. 6). Stage A is the initial morphogenetic stage before new neurons exhibit immature neuron-like morphology and involves polarization, migration, and the initial axonal and dendritic growth of these neurons. Stage B is the stage of further axonal and dendritic growth that sets up the basic organization for synaptic connections. During stage B, dendritic processes reach the outer edge of the molecular layer, and axon fibers reach the CA3 area. Spine growth takes place in stage $\mathrm{C}$, which also includes the later period of axonal and dendritic growth. Stage $\mathrm{C}$ is when the major synaptic connections are formed. Stage D describes further synaptic modifications, which are reflected in the formation of mushroom spines. Stage D begins shortly after spine formation during stage $\mathrm{C}$ and continues for several months.

\section{Temporal relationship between axonal-dendritic growth and spine formation}

Dendritic and axonal growth take place during stage $\mathrm{B}$. In the middle of stage $\mathrm{B}$, axon fibers reach $\mathrm{CA} 3$ and begin to interact with pyramidal neurons; therefore, we subdivided stage $B$ into $\mathrm{B} 1$ and B2. B1 covers the initial growth of axonal and dendritic processes. B2 starts when the axon fibers enter the CA3 area and ends 5-6 d later when spine growth starts (Fig. 6). The temporal relationship between axonal and spine growth in the adult mouse brain is consistent with findings in the postnatal rat brain. Retrograde tracing of the lipophilic dye DiI injected into the CA3 area revealed granule neurons in the dentate gyrus with immature dendritic arborization 2-3 d before the first spines were seen in these cells (Jones et al., 2003).

The transition from stage $\mathrm{B}$ to $\mathrm{C}$ might be associated with different mechanisms for cell survival by virtue of the initiation of connectivity. The successive interaction of newborn neurons with the hilar mossy cells and interneurons during B1, and with CA3 pyramidal neurons during B2, may contribute differentially to target-dependent cell survival, whereas afferent influences on cell survival might be more important after spine formation during stage C (Oppenheim, 1991).

\section{Delayed maturation of neurons in the adult dentate gyrus}

Consistent with electrophysiological studies (Liu et al., 1996; Ambrogini et al., 2004; Esposito et al., 2005), we found that newborn neurons in the adult and postnatal brains go through similar morphological transitions. In addition, they have similar patterns of dendritic and axonal outgrowth, indicating that the guidance cues for both processes are unaltered, possibly throughout the animals' life under normal conditions.

Compared with the early postnatal brain, the maturation of neurons in the adult is significantly delayed at the end of stage B2, when spines start to appear, which is correlated with a delay in dendritic growth. It is not clear, however, when this delay actually takes place. After a neural progenitor exits the cell cycle, it needs to differentiate, polarize, migrate into the granule cell layer, and start neurite outgrowth. All of these processes can be affected by changes in the extrinsic environment or intrinsic properties of immature cells. The local environments are dramatically different between the adult and 
developing brains. For example, the hippocampal circuitry is much more complex in the adult brain than in the developing brain. The properties of the astroglia in the hippocampus are also different between adult and early postnatal brains (Song et al., 2002). Furthermore, although they have not been described in the dentate gyrus, synchronous spontaneous activities exist in the CA1 and CA3 areas only during early postnatal development (Leinekugel et al., 1997; Garaschuk et al., 1998; Ben-Ari, 2001). These different properties between adult and postnatal brains may contribute to the different maturation rates that we see in the dentate granule neurons.

\section{Experience-dependent} neuronal maturation

Although spine growth reaches a plateau at $56 \mathrm{dpi}$, spine modification is more prolonged, which is reflected in the continuous increase in mushroom spine density (Fig. $6 b$ ). The change in mushroom spine density could be accounted for by two possible explanations: the mushroom spine density might increase gradually over a prolonged period of time, or new neurons with higher mushroom density might survive better than those with lower mushroom density. Studies that used BrdU labeling have suggested that most, if not all, cells persist if they survive after the first 4 weeks (Kempermann et al., 2003); therefore, it is more likely that mushroom density increases gradually over an extended period of time.

The prolonged structural modification in dendritic spines is accelerated by running. Indeed, running has an effect on dendritic spines at a very early stage, because spine motility is significantly higher in running mice at $21 \mathrm{dpi}$. Studies in visual and somatosensory cortices have shown that spine motility is downregulated by blocking visual and somatosensory input during the peak of synaptogenesis (Lendvai et al., 2000; Konur and Yuste, 2004) and is upregulated during the critical period of monocular deprivation (Majewska and Sur, 2003). Spine number did not change under these conditions, consistent with our finding that running does not change spine density. In addition, spine motility decreases during development (Dunaevsky et al., 1999), and it is inhibited by the activation of glutamate receptors (Fischer et al., 2000). These studies suggest that spine motility is regulated at least by two factors: synaptic input at the time of spine formation and the history of synaptic activity. Voluntary exercise in rodents can lead to an upregulation of genes related to neuronal activity and synaptic plasticity (Tong et al., 2001; Molteni et al., 2002; Farmer et al., 2004), which could be responsible for the high spine motility in running mice at $21 \mathrm{dpi}$, when the peak of spine growth occurs in new neurons. The fast reduction in spine motility in running mice indicates that the synapses formed on these spines are stabilized, which is correlated with the accelerated formation of mushroom spines.

Running mice learn better in the Morris water-maze test, a finding that has been correlated with the increase in the number of newborn neurons (van Praag et al., 1999). We show here that spine density does not change in running mice. Newborn neurons also have similar dendritic length and dendritic branching at 28 dpi under sedentary and running conditions (van Praag et al., 2005). The total number of new synapses formed in running mice is significantly higher because of the increase in the total number of newborn neurons. The increase in the potential of forming more synapses could contribute, in part, to the beneficial effect of running.

\section{Functional implications of the distinct morphological stages}

Studies attempting to selectively ablate adult neurogenesis in rats have suggested that new neurons at the age of 1-2 weeks are required for the hippocampus-dependent trace-conditioning task (Shors et al., 2001). We show here that spine growth starts at $\sim 16$ dpi in neurons born in the adult mouse brain (Fig. $6 b$ ), and it is similar in the adult rat brain (data not shown). This finding implies that newborn cells may be required for the formation and/or expression of trace memory even before they begin to form synapses on spines. A recent study has used localized irradiation to ablate neurogenesis in the adult rat brain (Snyder et al., 2005) and has indicated that 4- to 28 -d-old neurons are required for long-term memory. An important question raised by these studies is whether all neurons between 4 and $28 \mathrm{~d}$ of age, or rather neurons of specific ages within this time range, are required for the formation of long-term spatial memory (Snyder et al., 2005).

Although these studies need to be confirmed with more specific ways of ablating neurogenesis, they support the idea that new neurons might be functional at very early stages, before their final structural maturation. A distinct feature of newborn neurons in the adult brain is that they are structurally dynamic during their immature stages, before most of the afferent and efferent connections are 
made. Immature neurons have also been shown to have a lower threshold for long-term potentiation induction (Wang et al., 2000; Schmidt-Hieber et al., 2004). Currently, the precise age and function of the immature neuron that are important for memory formation are not known. Is the important time for memory formation the first 1-2 weeks, as studies by Shors et al. (2001) have suggested, or later, when most of spines are formed? Because the formation of dendritic spines is spatially constrained by the dendritic arborization, the early patterns of dendritic growth could restrict the later innervation of dendritic spines; however, specific synaptic input at the time of spine formation may have a greater impact on the specific connections formed on the new neurons. It is therefore likely that the afferent input to newborn neurons is determined by both dendritic arborization and spine formation. Future functional experiments with high spatial and temporal resolutions will be needed to pinpoint the time window when new neurons are most critical for hippocampusdependent functions.

\section{References}

Altman J, Das GD (1965) Autoradiographic and histological evidence of postnatal hippocampal neurogenesis in rats. J Comp Neurol 124:319-335.

Ambrogini P, Lattanzi D, Ciuffoli S, Agostini D, Bertini L, Stocchi V, Santi S, Cuppini R (2004) Morpho-functional characterization of neuronal cells at different stages of maturation in granule cell layer of adult rat dentate gyrus. Brain Res 1017:21-31.

Ben-Ari Y (2001) Developing networks play a similar melody. Trends Neurosci 24:353-360.

Bonhoeffer T, Yuste R (2002) Spine motility: phenomenology, mechanisms, and function. Neuron 35:1019-1027.

Dunaevsky A, Tashiro A, Majewska A, Mason C, Yuste R (1999) Developmental regulation of spine motility in the mammalian central nervous system. Proc Natl Acad Sci USA 96:13438-13443.

Dupret D, Montaron MF, Drapeau E, Aurousseau C, Le Moal M, Piazza PV, Abrous DN (2005) Methylazoxymethanol acetate does not fully block cell genesis in the young and aged dentate gyrus. Eur J Neurosci 22:778-783.

Eriksson PS, Perfilieva E, Bjork-Eriksson T, Alborn AM, Nordborg C, Peterson DA, Gage FH (1998) Neurogenesis in the adult human hippocampus. Nat Med 4:1313-1317.

Esposito MS, Piatti VC, Laplagne DA, Morgenstern NA, Ferrari CC, Pitossi FJ, Schinder AF (2005) Neuronal differentiation in the adult hippocampus recapitulates embryonic development. J Neurosci 25:10074-10086.

Farmer J, Zhao X, van Praag H, Wodtke K, Gage FH, Christie BR (2004) Effects of voluntary exercise on synaptic plasticity and gene expression in the dentate gyrus of adult male Sprague-Dawley rats in vivo. Neuroscience 124:71-79.

Fischer M, Kaech S, Wagner U, Brinkhaus H, Matus A (2000) Glutamate receptors regulate actin-based plasticity in dendritic spines. Nat Neurosci 3:887-894.

Garaschuk O, Hanse E, Konnerth A (1998) Developmental profile and synaptic origin of early network oscillations in the CA1 region of rat neonatal hippocampus. J Physiol (Lond) 507:219-236.

Gould E, Reeves AJ, Fallah M, Tanapat P, Gross CG, Fuchs E (1999) Hippocampal neurogenesis in adult Old World primates. Proc Natl Acad Sci USA 96:5263-5267.

Harris KM, Stevens JK (1989) Dendritic spines of CA1 pyramidal cells in the rat hippocampus: serial electron microscopy with reference to their biophysical characteristics. J Neurosci 9:2982-2997.

Hastings NB, Gould E (1999) Rapid extension of axons into the CA3 region by adult-generated granule cells. J Comp Neurol 413:146-154.

Jones EG, Powell TP (1969) Morphological variations in the dendritic spines of the neocortex. J Cell Sci 5:509-529.

Jones SP, Rahimi O, O’Boyle MP, Diaz DL, Claiborne BJ (2003) Maturation of granule cell dendrites after mossy fiber arrival in hippocampal field CA3. Hippocampus 13:413-427.

Kempermann G, Kuhn HG, Gage FH (1997) More hippocampal neurons in adult mice living in an enriched environment. Nature 386:493-495.

Kempermann G, Gast D, Kronenberg G, Yamaguchi M, Gage FH (2003) Early determination and long-term persistence of adult-generated new neurons in the hippocampus of mice. Development 130:391-399.

Konur S, Yuste R (2004) Developmental regulation of spine and filopodial motility in primary visual cortex: reduced effects of activity and sensory deprivation. J Neurobiol 59:236-246.

Kootstra NA, Matsumura R, Verma IM (2003) Efficient production of human FVIII in hemophilic mice using lentiviral vectors. Mol Ther 7:623-631.

Leinekugel X, Medina I, Khalilov I, Ben-Ari Y, Khazipov R (1997) $\mathrm{Ca}^{2+}$ oscillations mediated by the synergistic excitatory actions of GABA(A) and NMDA receptors in the neonatal hippocampus. Neuron 18:243-255.

Lendvai B, Stern EA, Chen B, Svoboda K (2000) Experience-dependent plasticity of dendritic spines in the developing rat barrel cortex in vivo. Nature 404:876-881.

Liu YB, Lio PA, Pasternak JF, Trommer BL (1996) Developmental changes in membrane properties and postsynaptic currents of granule cells in rat dentate gyrus. J Neurophysiol 76:1074-1088.

Majewska A, Sur M (2003) Motility of dendritic spines in visual cortex in vivo: changes during the critical period and effects of visual deprivation. Proc Natl Acad Sci USA 100:16024-16029.

Markakis EA, Gage FH (1999) Adult-generated neurons in the dentate gyrus send axonal projections to field CA3 and are surrounded by synaptic vesicles. J Comp Neurol 406:449-460.

Matsuzaki M, Ellis-Davies GC, Nemoto T, Miyashita Y, Iino M, Kasai H (2001) Dendritic spine geometry is critical for AMPA receptor expression in hippocampal CA1 pyramidal neurons. Nat Neurosci 4:1086-1092.

Molteni R, Ying Z, Gomez-Pinilla F (2002) Differential effects of acute and chronic exercise on plasticity-related genes in the rat hippocampus revealed by microarray. Eur J Neurosci 16:1107-1116.

Naviaux RK, Costanzi E, Haas M, Verma IM (1996) The pCL vector system: rapid production of helper-free, high-titer, recombinant retroviruses. J Virol 70:5701-5705.

Nimchinsky EA, Sabatini BL, Svoboda K (2002) Structure and function of dendritic spines. Annu Rev Physiol 64:313-353.

Oppenheim RW (1991) Cell death during development of the nervous system. Annu Rev Neurosci 14:453-501.

Overstreet LS, Hentges ST, Bumaschny VF, de Souza FS, Smart JL, Santangelo AM, Low MJ, Westbrook GL, Rubinstein M (2004) A transgenic marker for newly born granule cells in dentate gyrus. J Neurosci 24:3251-3259.

Peters A, Kaiserman-Abramof IR (1970) The small pyramidal neuron of the rat cerebral cortex: the perikaryon, dendrites and spines. Am J Anat 127:321-355.

Sala C (2002) Molecular regulation of dendritic spine shape and function. Neurosignals 11:213-223.

Schikorski T, Stevens CF (1997) Quantitative ultrastructural analysis of hippocampal excitatory synapses. J Neurosci 17:5858-5867.

Schmidt-Hieber C, Jonas P, Bischofberger J (2004) Enhanced synaptic plasticity in newly generated granule cells of the adult hippocampus. Nature 429:184-187.

Shors TJ, Miesegaes G, Beylin A, Zhao M, Rydel T, Gould E (2001) Neurogenesis in the adult is involved in the formation of trace memories. Nature 410:372-376.

Snyder JS, Hong NS, McDonald RJ, Wojtowicz JM (2005) A role for adult neurogenesis in spatial long-term memory. Neuroscience 130:843-852.

Song H, Stevens CF, Gage FH (2002) Astroglia induce neurogenesis from adult neural stem cells. Nature 417:39-44.

Squire LR, Zola-Morgan S (1991) The medial temporal lobe memory system. Science 253:1380-1386.

Tong L, Shen H, Perreau VM, Balazs R, Cotman CW (2001) Effects of exercise on gene-expression profile in the rat hippocampus. Neurobiol Dis 8:1046-1056.

van Praag H, Christie BR, Sejnowski TJ, Gage FH (1999) Running enhances neurogenesis, learning, and long-term potentiation in mice. Proc Natl Acad Sci USA 96:13427-13431.

van Praag H, Schinder AF, Christie BR, Toni N, Palmer TD, Gage FH (2002) Functional neurogenesis in the adult hippocampus. Nature 415:1030-1034.

van Praag H, Shubert T, Zhao C, Gage FH (2005) Exercise enhances learning and hippocampal neurogenesis in aged mice. J Neurosci 25:8680-8685.

Wang LP, Kempermann G, Kettenmann H (2005) A subpopulation of precursor cells in the mouse dentate gyrus receives synaptic GABAergic input. Mol Cell Neurosci 29:181-189.

Wang S, Scott BW, Wojtowicz JM (2000) Heterogeneous properties of dentate granule neurons in the adult rat. J Neurobiol 42:248-257.

Zufferey R, Donello JE, Trono D, Hope TJ (1999) Woodchuck hepatitis virus posttranscriptional regulatory element enhances expression of transgenes delivered by retroviral vectors. J Virol 73:2886-2892. 